\title{
Multi-sensor System For Indoor Environment Monitoring
}

\author{
Zheng-yu Wang ${ }^{a}$, and Xiao-ru Zhang ${ }^{b}$ \\ Harbin Institute of Technology, Shenzhen 518000, China; \\ a313564106@qq.com, ${ }^{\mathrm{b}}$ 740211927@qq.com
}

\begin{abstract}
This paper designed an indoor environment monitoring system, in order to enhance the quality of indoor air. This system can accurately measure the temperature, humidity and the concentration of oxygen, carbon dioxide, carbon monoxide, formaldehyde, PM2.5 and PM10 using the sensor array and transfer the data to the mobile terminal through a wireless network. The system is implemented on ARM which is regarded as the core controller and integrates a number of functional modules. We select the sensors that have the characteristics of high sensitivity and robustness to carry out a real-time measurement. Data collected can be displayed either on the LCD or on the computer or phone via Wi-Fi. The system is not only easy to operate, but also has high stability, and the accuracy of it meet the needs of measurement. Our system can provide accurate and timely health reference to users.
\end{abstract}

Keywords: Environmental monitoring system, Multi-sensor; STM32F4, Wi-Fi, Interface design

\section{Introduction}

An important goal of smart home automation is to improve the user's comfort and security along with a reduced overall energy consumption. A core element of this strategy is an automated indoor climate control system [1]. With the improvement of public quality of life, people increasingly concerned about the quality of indoor air. Indoor air quality (IAQ) is a term referring to the air quality within and around buildings and structures, especially as it relates to the health and comfort of building occupants [2].

At present, the source of indoor pollutants is mainly included: kitchen cooking, indoor smoking which produced carbon monoxide and carbon dioxide. Formaldehyde and toluene produced by interior decoration [3]. Nowadays, most of the indoor environmental monitoring systems in the domestic market can only detect single pollution factor, such as formaldehyde detector, dust detector and most of the existing air quality measurement devices are designed for professionals. Such systems are expensive and beyond the reach of average users. Meanwhile poor air quality is extremely difficult for human beings to feel or sense, thus, most people cannot tell whether or not indoor air quality is bad [4]. Therefore developing a low-cost but high-performance indoor environment monitoring system is imminent.

In this paper, we present a precise wireless multi-sensor network system and it integrated various types of sensor modules, alarm module, display module, wireless transmission module and so on. System achieve a human-computer interaction interface through the interface programming and multi-threaded programming. Greatly enhance the air quality of indoor environment .

The rest of the paper is organized as follows. In next sections, the architecture of overall system is described. Then, the hardware and software design are presented in details. Finally, some experimental measurement results are presented and a summary is included in the end.

\section{System Framework}

In this paper, the overall framework of the indoor environment monitoring system shown in Figure 1, this system will select the 32 bit ARM Cortex-m4 core STM32F407 microcontroller as the main control chip, and integrate the sensor module, display module, wireless transmission module and so on. The sensor module consists of temperature and humidity sensor, formaldehyde sensor, PM2.5, PM10 sensor, carbon monoxide sensor, carbon dioxide sensor. Display module is an onboard 
3.7-inch LCD which was exchanged data with and development board through the FSMC interface. Wireless communication uses the ESP8266 module. When the host computer connected to the AP hot spots, users can see the concentration value of the various indoor gas from phone or computer.

Here we present the working principle of the system: When the ARM connected to the power supply, the sensor began to work. Through the serial interrupt, the sensor sent the data to the development board, and then the data that was processed will be displayed on the LCD. If the concentration value of some type of pollutant is abnormal, higher than the standard value, the signal will trigger the alarm module. Processed data will be uploaded to the mobile terminal through the wireless module. When the indoor environment monitoring interface was opened, user can observe the indoor air condition in real time. Then, the user can make the appropriate treatment to improve the indoor environment quality.

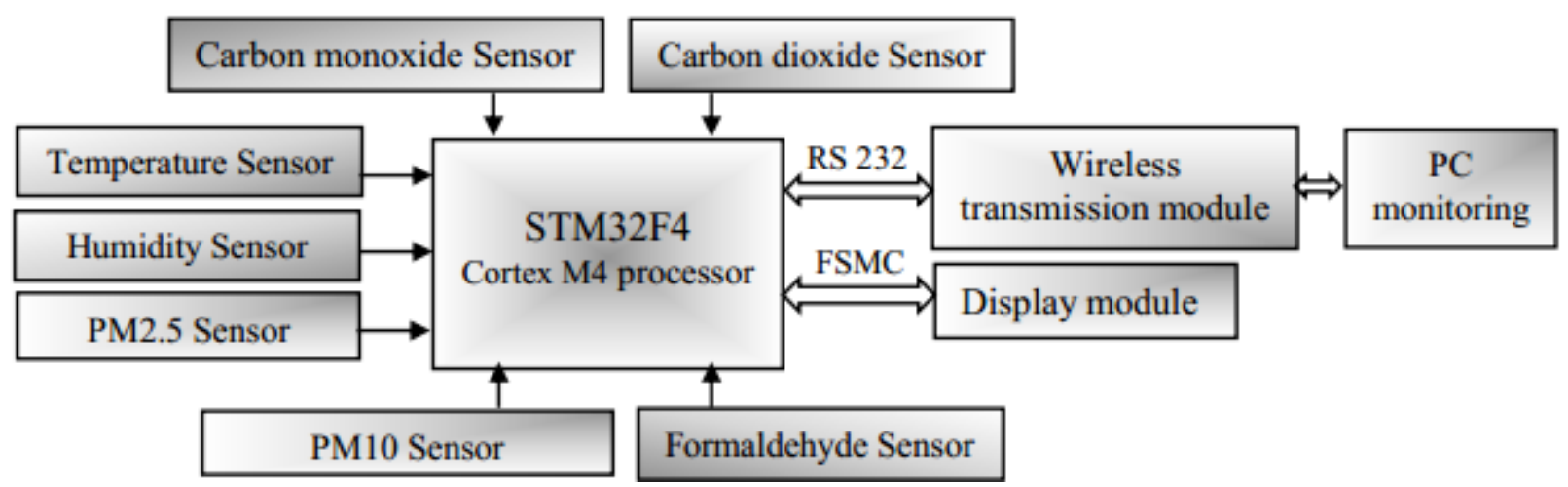

Fig. 1 Block diagram of the environmental monitoring system

\section{System Hardware Design}

\subsection{Core processor}

In order to achieve the low power consumption, faster processing speed, rich peripherals and other requirements, the system uses the STM32F407ZGT6 chip which based on the Cortex M4 core architecture. The main frequency of the development board is up to $168 \mathrm{MHz}$, which achieve the requirements of rapid processing. The development board has a function of real-time clock (RTC), and its IO multiplexing function greatly enhanced. Data reading become more flexible, and per megabits of $238 \mathrm{uA}$ power consumption makes the system meet the requirements of low power consumption.

\subsection{Sensor introduction.}

The sensor is the terminal node of the system, its quality directly determines the merits of the whole system. The model of temperature and humidity sensor is DHT11, this sensor has the advantages of high stability and low power consumption, and is responsible for collecting the data of indoor thermal environment. The sensor exchange data with ARM via a single bus.

The model of the formaldehyde sensor is DS-HCHO, this sensor has high resolution, and its digital transmission is stable, using electrochemical principles for gas detection.

The model of carbon monoxide sensor is ME2-CO, this sensor has the advantages of high sensitivity, anti-interference ability and long life. Formaldehyde and carbon monoxide sensor communicate with the microcontroller through the serial port.

Detection of PM2.5 and PM10 use high-precision laser sensor which is highly integrated. This sensor has quick response for scene switching, and uses laser scattering principle to detect the diameter of the particles. When the suspended particles in the air was inhaled into the light sensitive area by the built-in fan, this will make the laser scattering. Light-sensitive devices detect the scattered light signal, and output the voltage signal which is proportional to the light intensity. Through the analog-to-digital converter, the analog signal changed into digital signal which will be sent to the microprocessor. The internal schematic diagram of sensor is shown in Figure 2. 
Detection of carbon dioxide uses the COZIR-W which was produced by the UK. The warm-up time of this sensor is short, and the power consumption of it is low. This sensor uses non-dispersive infrared technology for measurement, which can obtain accurate and stable data.

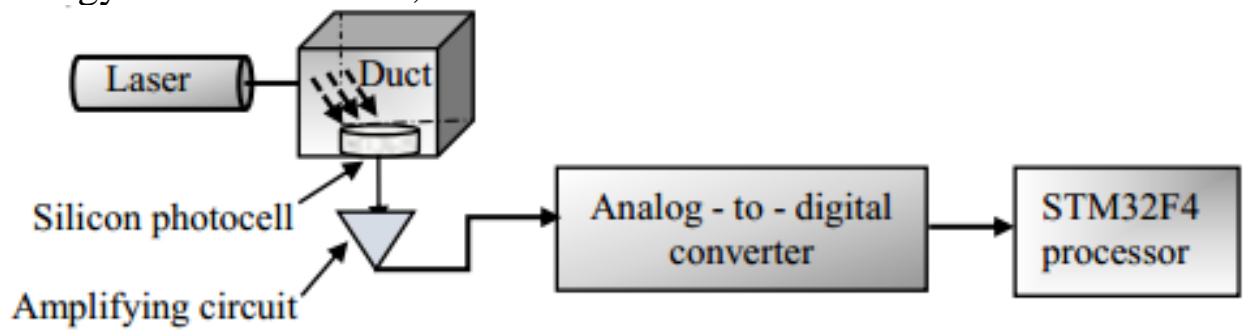

Fig. 2 PM2.5 sensor internal structure

\subsection{WiFi introduction.}

The Model of WiFi is ESP8266, and its core is a diamond standard 106 microcontroller, a low-power 32-bit RISC controller. The module supports a complete TCP / IP protocol stack. Users can use this module to add networking capabilities to existing devices, or to build standalone network controllers. The model supports AP (Access Point), STA (Station), AP + STA coexistence mode, and use efficient AT command. In this system, the WiFi module communicates with ARM through the serial port.

\section{System Software Design}

\subsection{Sensor control instructions.}

Although the individual sensor has low power consumption, all these sensors working for a long time can produce huge energy consumption. Therefore, designing an efficient work procedure becomes the key to the problem. Meanwhile, every sensor has its own life, if we used it improperly, the life of it will be less. To overcome this limitation for practical use, correction algorithms are successfully applied and the life time could be extended to several years $[5,6]$. The procedure make the sensor periodically into the sleep, wake up, and work state, not only reduce the power greatly, but also complete the detection task effectively. Developer programmed to set the operating cycle, when the sensor outside the operating cycle, it will into sleep state. When the sensor enters the duty cycle, it will be waken up. Meanwhile, the central processing unit which is on the development board sends a command to the sensor, as a response, the sensor returns the data to the development board. Once the abnormal data is found, the development board will trigger the buzzer alarm. The working flow char is shown in Figure 3.

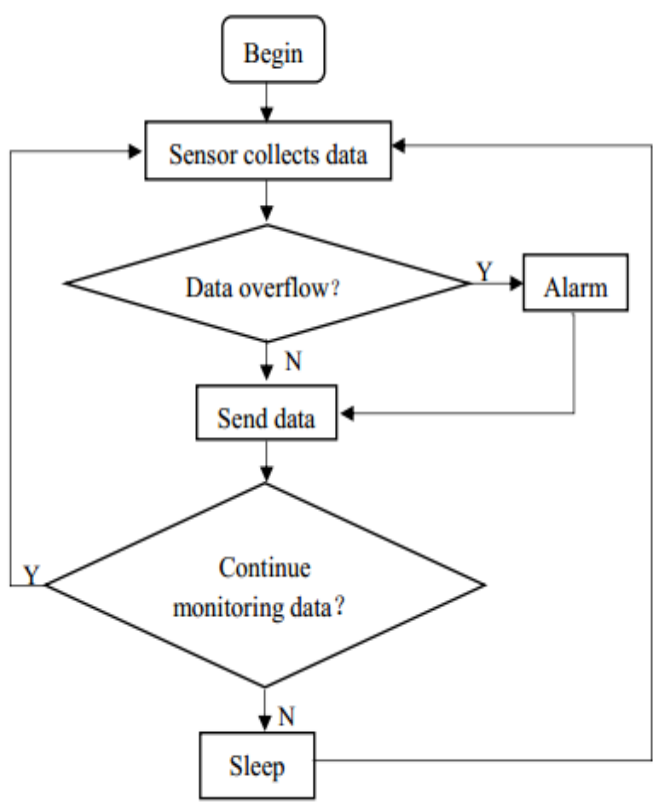

Fig. 3 Sensor workflow 


\subsection{WiFi control instructions.}

The system uses wireless communication module, through the AT command user can control it. The module works in the AP mode, and acts as a wifi hotspot, allowing the mobile terminal to connect. The AT commands are shown in the table below.

Table 1 AP mode instructions

\begin{tabular}{cc}
\hline Send instructions & effect \\
\hline AT+CWMODE & Set AP mode \\
AT+RST & Restart effective \\
AT+CWSAP=”ATK-ESP8266”,"12345678, 1,4 & Set AP paramaeters \\
AT+CIPMUX=1 & Open multiple connections \\
AT+CIPSERVER=1,8086 & Open server mode, set the port to 8086 \\
AT+CIPSEND $=0, n$ & Send n bytes of data \\
\hline
\end{tabular}

\subsection{Socket design.}

The network communication in this system is based on the client / server model. In the TCP / IP network, C/S model has two main characteristics, one of them is the non-reciprocal of the host on the network and another is asynchronous communication operations. The socket encapsulates the transport protocol and realizes the data transmission in the different network processes. The IP address and port number of the host are called socket addresses. Using socket to complete the data transmission requires three procedures. Firstly, create and open the socket. Secondly, send and receive data to the socket. Finally, close the socket. In order to meet the real-time transmission, the accuracy of the data and other requirements, the system uses streaming socket.

\subsection{System interface design.}

The Programming of human-computer interface is based on the VS2010. The interface includes five parts such as the main interface, the registration interface, the login interface, the data interface and so on. Each interface uses a different thread in order to make the code more efficient.

\section{System Test}

Based on the design of hardware and software above, we designed a portable monitor as shown in Figure 4.

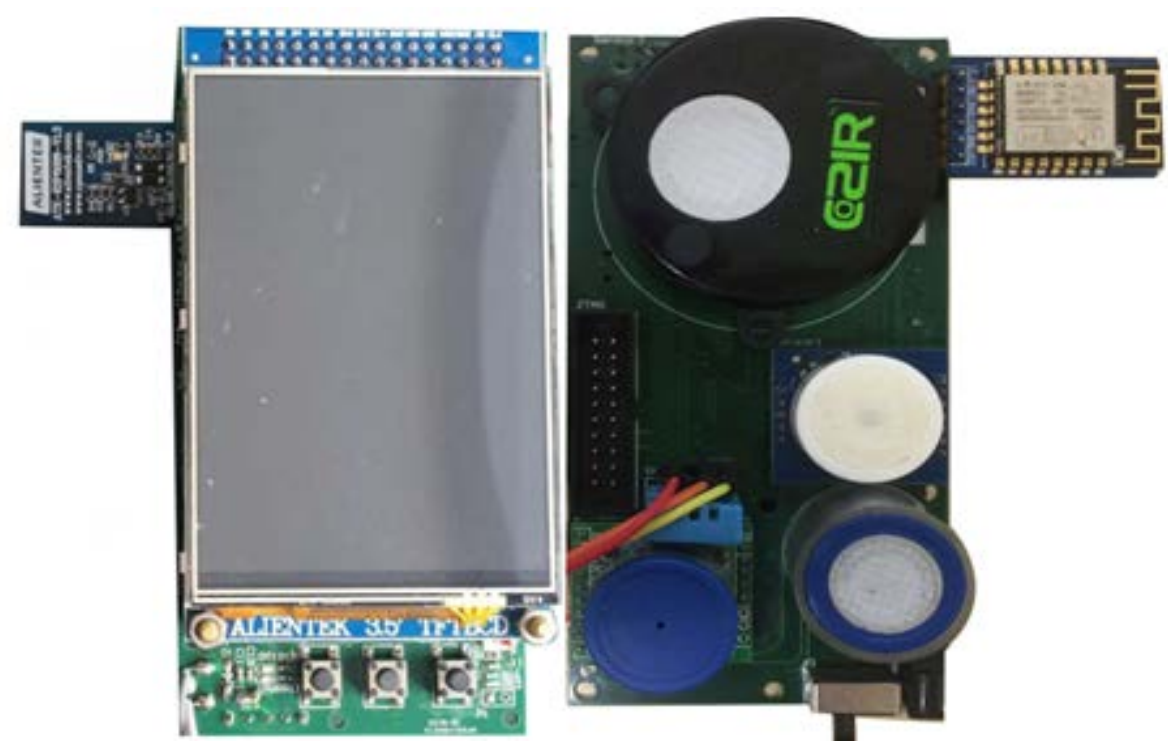

Fig. 4 Own production of portable detectors

Put the monitor in the bedroom, and record the data of temperature and humidity throughout the day. In order to make the measurement results more accurate, we calculated the average of the data recorded within each hour. The test results are compared with the standard hygrometer, the 
comparing results are shown in figure 5 and figure 6. As we can see, there is an acceptable error between the measured value and the actual value.

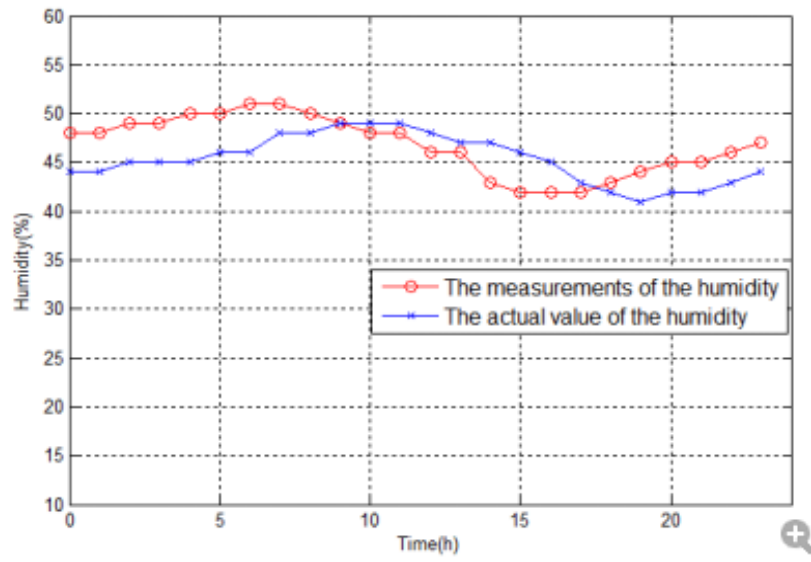

Fig. 5 Temperature contrast

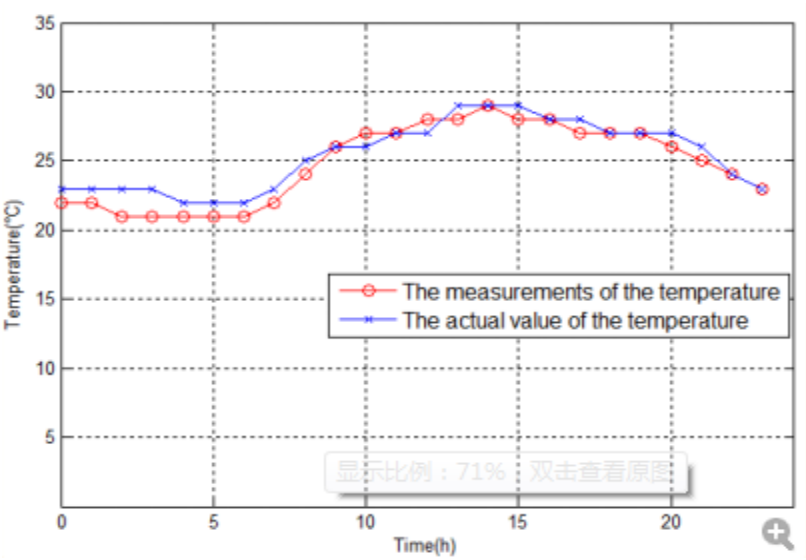

Fig. 6 Humidity contrast

We use smoke to check the accuracy of CO2, CO, HCHO and PM2.5 sensors. Place burning cigarettes into a container, and then we put our monitor and the standard detectors into the box at the same time. In order to ensure the reliability of the data, We measured twenty sets of data and the values in each set were averaged over a 4-second basis. The measurement results were shown in figure 7 to figure 10. Compare the measurement results, we can find that there is a deviation, but it is within the allowable range of error. When the smoke increased, the concentration of the detected gas greatly improved. When the concentration exceed the safety limit, the buzzer alarm will be triggered.

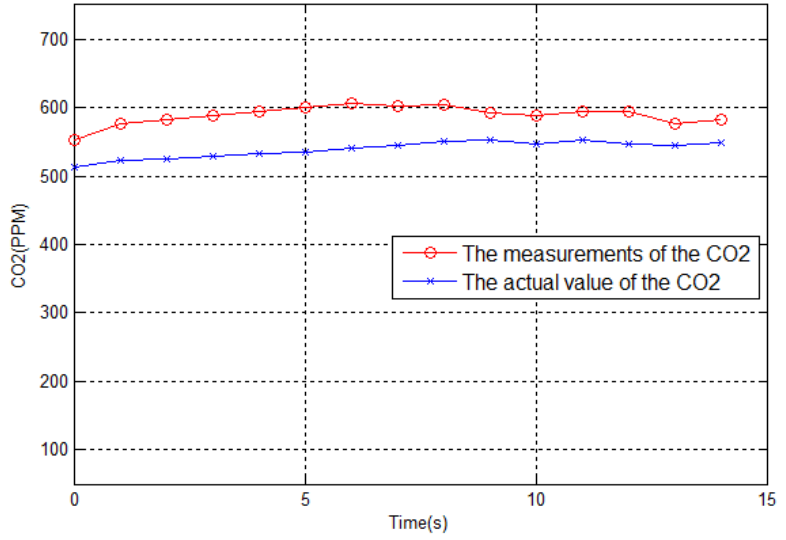

Fig. 7 CO2 contrast

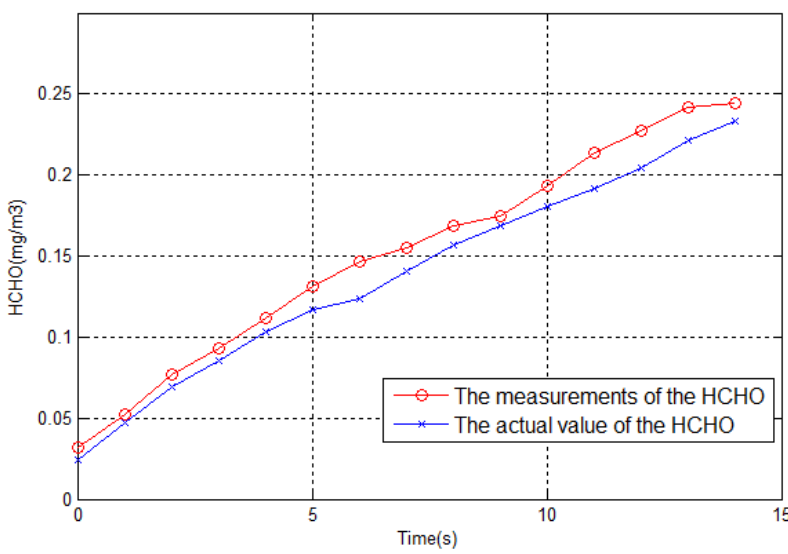

Fig. 9 HCHO contrast

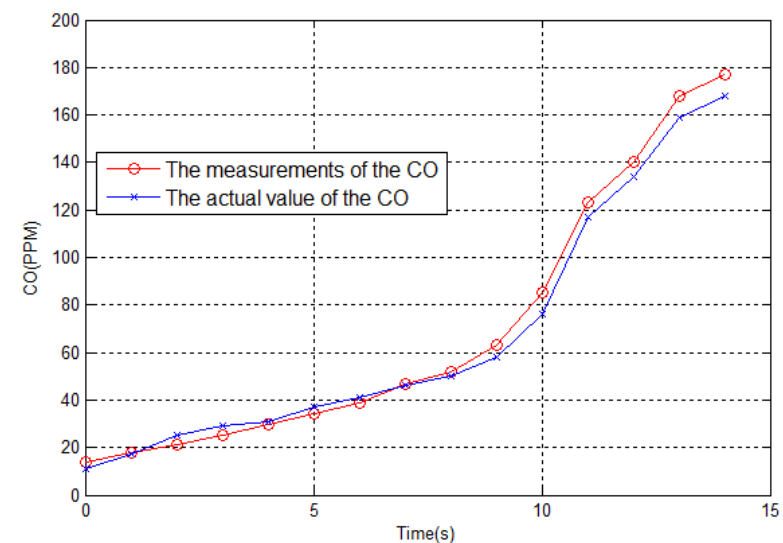

Fig. 8 CO contrast

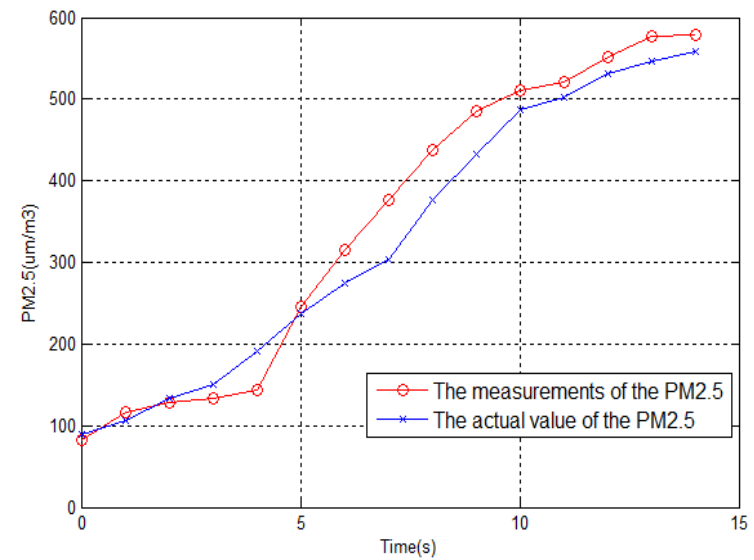

Fig. 10 PM2.5 contrast

Through the comparison, we can conclude that the accuracy of our indoor environment monitoring system is basically consistent with the single detector and satisfies the actual testing requirements. At 
the same time, the design not only make up for the lack of a single detection device, but also add the new function of remote monitoring, greatly enhance the practicality.

\section{Conclusions}

In view of the shortcomings and deficiencies of the environment detector in current market. We designed a portable indoor environment monitor based on Wi-Fi transmission. Not only meet the requirements of measuring a variety of pollutants at the same time, but also achieve the purpose of remote detection of indoor environment. The product is still in the testing stage, and we will encounter many unknown problems in the future. Therefore, improving the stability of the program and optimizing the hardware structure are the focus in the future study.

\section{Acknowledgments}

This work was supported by Basic layout of Shenzhen City (no.JCYJ20150827165024088), Supporting platform project in Guangdong Province (no. 2014B0909B001).

\section{References}

[1] Ivanov B, Zhelondz O, Borodulkin L, et al. Distributed smart sensor system for indoor climate monitoring[J]. Konnex Scientific Conf, 2002:10-11.

[2] Yao D J. A gas sensing system for indoor air quality control and polluted environmental monitoring[C]// Nanotechnology, 2009. Ieee-Nano 2009. IEEE Conference on. IEEE, 2009:806-811.

[3] Abraham S, Li X. Design of A Low-Cost Wireless Indoor Air Quality Sensor Network System[J]. International Journal of Wireless Information Networks, 2016, 23(1):57-65.

[4] Abraham S, Li X. A Cost-effective Wireless Sensor Network System for Indoor Air Quality Monitoring Applications [J]. Procedia Computer Science, 2014, 34:165-171.

[5] Datasheet CO2 Sensor, SBU Schirmer + Berthold Umwelttechnik GmbH, 1999

[6] Datasheet Sensoric CO 3E300, H2S E50, H2 E2000, O3 2E1: Unitronic GmbH, 1998 